\title{
Trait Anxiety Moderates the Relationship Between Testosterone Responses to Competition and Aggressive Behavior
}

\author{
Rachel E. Norman • Benjamin J. P. Moreau • \\ Keith M. Welker • Justin M. Carré
}

Received: 2 September 2014 / Revised: 2 November 2014 / Accepted: 6 November 2014 /

Published online: 18 November 2014

(C) Springer International Publishing 2014

\begin{abstract}
Testosterone (T) concentrations change rapidly in the context of human competition, and these changes in neuroendocrine function may serve to modulate future aggressive behavior. However, an increase in $\mathrm{T}$ during competition does not translate into aggressive behavior among all individuals. Here, we examined the extent to which individual differences in trait anxiety moderate the relationship between $\mathrm{T}$ responses to competition and aggressive behavior. Across two studies, we found that $T$ responses to competition were positively correlated with subsequent aggression, but only among men scoring relatively low in trait anxiety. Trait anxiety did not moderate the relationship between $\mathrm{T}$ reactivity and aggression in women. These findings highlight the importance of considering individual difference in trait anxiety when examining the neuroendocrine correlates of human aggression.
\end{abstract}

Keywords Testosterone $\cdot$ Human competition · Trait anxiety · Dominance - Aggression

\section{Introduction}

Testosterone ( $\mathrm{T}$ ), a steroid hormone produced and secreted primarily by the gonads, plays an important role in modulating physiological and behavioral processes of relevance to survival and reproduction (Ketterson and Nolan 1992). In animal models, a large body of evidence has linked T to aggressive behavior (see Simon and Lu 2006). In contrast, individual differences in baseline $\mathrm{T}$ concentrations are only weakly correlated with various measures of aggression in humans ( $r=.08$, see Archer et al. 2005 for meta-analysis).

R. E. Norman • B. J. P. Moreau • J. M. Carré $(\bowtie)$

Department of Psychology, Nipissing University, North Bay, ON, Canada

e-mail: justinca@nipissingu.ca

K. M. Welker

Department of Psychology, University of Colorado Boulder, Boulder, CO, USA 
Importantly, $\mathrm{T}$ concentrations are not static, but rather, fluctuate rapidly in the context of competitive interactions (Wingfield et al. 1990; Oliveira 2009; Archer 2006), prompting researchers to suggest that perhaps T reactivity to social threat, rather than baseline $\mathrm{T}$ concentrations, may be more relevant to individual differences in aggressive behavior (McGlothlin et al. 2007). Consistent with this idea, a growing number of studies have found that $\mathrm{T}$ responses to competitive interactions predict subsequent aggression (Carré et al. 2009, 2013, 2014a) and antagonistic behavior (Geniole et al. 2013). These correlational studies provide support for the idea that acute changes in T during competition may serve to fine-tune dominance-related behavior (Mazur 1985). More compelling support for this idea comes from work with male California mice in which $\mathrm{T}$ administration to winners of a competitive interaction causally modulates subsequent aggressive behavior and probability of winning (Fuxjager et al. 2010; Gleason et al. 2009; Trainor et al. 2004).

Research in men indicates that changes in $\mathrm{T}$ during a competitive interaction predict subsequent aggressive behavior, with effect sizes ranging from moderate to large ( $r$ values between .26 and .45; Carré et al. 2009; 2013). Despite the impressive effect sizes for the relationship between $\mathrm{T}$ reactivity and aggression, there may be some individuals for whom $T$ reactivity has stronger (or weaker) effects on aggression, and thus, studies investigating potential moderators of the T-aggression relationship are warranted (Carré et al. 2011). For instance, one study reported that winners who increased in T engaged in heightened aggressive behavior, but only to the extent that they also scored high on a self-report measure of trait dominance (Carré et al. 2009). Trait anxiety, a measure of one's tendency to perceive situations as threatening, is one prime candidate for a moderator of the T-aggression relationship. The possibility that trait anxiety moderates the relationship between $\mathrm{T}$ dynamics and aggression is suggested by work in animal models and in humans. For instance, Veenema et al. (2007) found that rats selectively bred for low levels of anxiety demonstrate increased $\mathrm{T}$ reactivity and heightened aggressive behavior during a resident intruder paradigm relative to rats selectively bred for high levels of anxiety. Work in humans indicates that losers of competition demonstrate a sharp decline in $\mathrm{T}$ relative to winners, but only to the extent that they score high on a measure of social anxiety (Maner et al. 2008). Also, other work indicates that trait anxiety moderates the effect of power priming on subsequent risk-taking and mate-seeking behavior (Maner et al. 2012). Specifically, it was found that priming people for power increased subsequent risk-taking and mate-seeking, but only among individuals scoring relatively low on trait anxiety (Maner et al. 2012). This finding is particularly noteworthy given that other work indicates that $\mathrm{T}$ responses to competition predict subsequent risk-taking behavior in men (Apicella et al. 2014).

Here, across two studies, we examined the extent to which trait anxiety moderates the relationship between $\mathrm{T}$ responses to competition and aggressive behavior. We hypothesized that an increase in T during a competition would be positively correlated with subsequent aggressive behavior, but only among individuals with low trait anxiety (Study 1). Moreover, we examined whether the moderating effect of trait anxiety would be similar in men and women (Study 2) and whether winning or losing a competition would further moderate the effect of trait anxiety on the relationship between $\mathrm{T}$ reactivity and aggression (i.e., 3-way interaction; Study 2). 


\section{Study 1}

\section{Materials and Methods}

\section{Participants}

80 male participants $\left(M_{\mathrm{age}}=21.58, S D=3.16\right)$ were recruited from the Nipissing University (NU) Research Participation Pool and from ads placed in the NU community. Participants received either course credit and a $\$ 5$ honorarium or $\$ 15$. Aggression data from two participants were lost due to computer malfunction.

\section{Procedure}

Participants first completed a consent form, after which they completed a small battery of self-report measures including the trait anxiety scale. In this study, we used the trait anxiety subscale of the State-Trait Anxiety Inventory (STAI) (Spielberger et al. 1983) to assess participants' general level of anxiety. The STAI included 20 items (e.g., "I feel nervous and restless", "I worry too much over something that really doesn't matter", "I am calm, cool, and collected [reverse scored]). Responses were recorded on 4-point Likert scale $(1=$ almost never, $4=$ almost always $)$. Trait anxiety scores were calculated by summing responses across items and dividing by 20 to obtain a measure of mean anxiety (Cronbach's $\alpha=.93, M=1.97, S D=.52$ ).

Next, a 1-2 mL saliva sample (pre-competition) was collected via passive drool into a polystyrene culture tube. Participants then played boxing on an Xbox 360 Kinect video game for $15 \mathrm{~min}$. Unbeknownst to participants, the difficulty level was set to low and thus they experienced a string of victories. Afterwards, participants completed a questionnaire assessing their thoughts about the video game ("how hard did you try to win this game?", "how frustrating did you find this game?") followed by a second, post competition, saliva sample obtained approximately $20 \mathrm{~min}$ after the onset of the video game competition. Saliva samples were stored at $-60 \mathrm{C}$ until assayed in house using commercially available enzyme immunoassay kits (DRG International). All samples were assayed in duplicate and the intra-assay CV was $5.67 \%$. Across high and low controls, the average inter-assay CV was $11.14 \%$.

As part of a broader research protocol examining the effects of self-control on aggressive behavior, participants were then randomly assigned to one of two conditions: self-control depletion or control condition. In both conditions, participants watched a short silent video clip (6 min) of a woman being interviewed. The woman was shown in the top left portion of the screen, and in the bottom right portion a series of one-syllable words (e.g. tree) would appear for $10 \mathrm{~s}$ each. In the depletion condition, participants were told not to read or look at any words that appeared on the screen. The control group was not given additional instructions other than to watch the video. Previous work using the same task reported that individuals in the depletion condition were more aggressive in a subsequent aggression task (DeWall et al. 2007).

Next, participants performed the Point Subtraction Aggression Paradigm (PSAP), a well-validated behavioral measure of reactive aggression (see Cherek et al. 2006 for review) where they were paired with a male partner (actually a computer program). A male confederate was present in the adjoining room to make the scenario more 
believable. The goal of the task was to earn as many points as possible, which would later be exchanged for money. Three response options were available: pressing number 1 a hundred consecutive times earned a point, pressing number 2 would steal a point from their partner after ten consecutive presses, and pressing number 3 protected their points from being stolen for a variable amount of time after ten presses. Number 2 button presses are considered a form of aggressive behavior (see Cherek et al. 2006) as stealing points is a behavioral strategy directed toward another individual with the purpose of harming them (financially). The game is structured to randomly deduct points, and this was attributed to their partner stealing points. Participants were instructed that they had been randomly assigned to an experimental condition whereby they would not get to keep stolen points even though their partner would lose the points. Participants and their partner would be paid based on the number of points they had accumulated during the $10 \mathrm{~min}$ long task. Consistent with our recent work (Carré et al. 2013), we computed aggressive behavior by regressing average aggressive responses onto average reward and average protection responses and saving the unstandardized residuals. This procedure removes variance in aggression that is explained by reward and protection responses, enabling us to investigate how much of the remaining variance in aggression is explained by trait anxiety, $\mathrm{T}$ reactivity, and the interaction between these variables.

\section{Analysis}

We first examined the extent to which experimental group (depletion vs. control) would be associated with aggressive behavior and/or would interact with the independent variables ( $T$ reactivity and trait anxiety). In accordance with other human research examining competition-induced T dynamics (e.g., Mehta and Josephs 2006; Carré et al. 2009) T reactivity was measured by regressing post-video game $T$ concentrations onto pre-video game T concentrations and saving the unstandardized residuals. Regression analyses indicated that experimental condition did not predict aggression $(p=.94)$ and there were no experimental condition X T reactivity $(p=.93)$, experimental condition $\mathrm{X}$ trait anxiety $(p=.94)$ or experimental condition X T reactivity $\mathrm{x}$ trait anxiety interactions $(p=.40)$. Thus, given that experimental condition had no effects on aggressive behavior and did not interact with our predictor variables, our primary analysis collapsed across experimental condition.

In order to examine the extent to which trait anxiety would moderate the relationship between $T$ reactivity and aggressive behavior, moderated multiple regression analysis was performed using the PROCESS macro for SPSS (Hayes 2012; 2013; Model 1), where residualized $\mathrm{T}$ change was set as the IV, trait anxiety as the moderator, and aggression residuals as the DV in Model 1. All predictors were mean-centered prior to computing the interaction term and simple slopes were calculated at \pm 1 SDs.

\section{Result and Discussion}

There were no bivariate correlations between $\mathrm{T}$ residuals and aggression $(r=-.001$, $p=.99)$, T residuals and trait anxiety $(r=.07, p=.57)$ or trait anxiety and aggression $(r=-.12, p=.31)$. Regression analyses confirmed that $\mathrm{T}$ reactivity and trait anxiety did 
not predict aggressive behavior $(B=-.06, S E=.68, p=.93,95 \%$ CIs $=-1.41$, $1.30, \mathrm{r}_{\text {partial }}=.00$ and $B=-34.66, S E=32.81, p=.29,95 \% \mathrm{CIs}=-100.04,30.73$, $\mathrm{r}_{\text {partial }}=-.12$, respectively). Nevertheless, consistent with our hypothesis, there was a significant $\mathrm{T}$ reactivity $\mathrm{X}$ trait anxiety interaction $(B=-3.38, S E=1.63$, $\left.p=.041,95 \% \mathrm{CIs}=-6.62,-.14, \mathrm{r}_{\text {partial }}=-.18\right)$. Simple slopes analyses indicated that $\mathrm{T}$ reactivity was positively, marginally associated with aggression in men with low trait anxiety $(-1 \mathrm{SD} ; B=1.72, S E=.997, p=.090,95 \% \mathrm{CIs}=-.27,3.7)$ but not associated with aggression in men with high trait anxiety $(+1 \mathrm{SD} ; B=-1.83, S E=1.18$, $p=.12,95 \% \mathrm{CIs}=-4.17, .51)$. The interaction term indicates that these two slopes significantly differed from each other. Moreover, individuals with high $\mathrm{T}$ reactivity and low anxiety displayed the most aggressive behavior. See Fig. 1 and Tables 1 and 2.

In summary, findings from Study 1 support our hypothesis that trait anxiety moderates the effect of $\mathrm{T}$ reactivity on subsequent aggression. Specifically, T reactivity is positively correlated with aggressive behavior, but only in men with relatively low trait anxiety. The relationship between $\mathrm{T}$ reactivity and aggression is in the opposite direction for men with relatively high anxiety. Thus, a rise in T appears to modulate aggressive behavior in low anxiety men, and submissive behavior in high anxiety men. To examine the robustness of this $\mathrm{T}$ reactivity-X-trait anxiety interaction, we reanalyzed results from an archival dataset (Carré et al. 2013). This dataset included both men and women, and also random assignment to win and loss conditions, enabling us to examine whether there are sex differences in the $\mathrm{T}$ reactivity-X-trait anxiety effect and also whether winning or losing further moderates this effect. In addition, other work suggests that trait anxiety moderates the effect of competition outcome on T reactivity in men. Specifically, losing was associated with a decrease in $\mathrm{T}$ concentrations relative to winning, but only in men scoring relatively high on a measure of social anxiety (Maner et al. 2008). A major limitation of this study is that the sample consisted of only 23 men. Here, we improve upon the study of Maner et al. (2008) by assessing the moderating effect of trait anxiety on the relationship between competition outcome and $\mathrm{T}$ reactivity. Moreover, we examined whether the trait anxiety-X-T reactivity interaction observed in Study 1 would extend to women, and whether the effect is further moderated by competition outcome. Some previous work suggests that $\mathrm{T}$ responses to competition predict future competitive and aggressive behavior in losers,

Fig. 1 Trait anxiety moderates the relationship between testosterone reactivity and aggressive behavior in men (Study 1). Note: Testosterone and trait anxiety are plotted at $+/-1$ SD from the mean

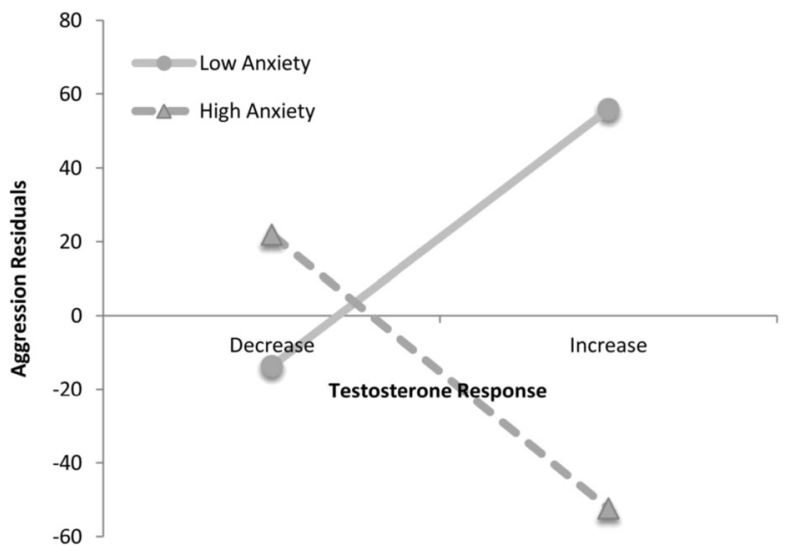


Table 1 Descriptive statistics for testosterone in Study 1

\begin{tabular}{llll}
\hline & Time & \multicolumn{2}{l}{ Winners } \\
\cline { 3 - 4 } & & $\mathrm{M}$ & \\
\cline { 3 - 4 } & & & SD \\
\hline \multirow{2}{*}{ Men } & 1 & 82.33 & 29.39 \\
& 2 & 79.45 & 29.50 \\
\hline
\end{tabular}

but not winners (Mehta and Josephs 2006; Carré et al. 2009), suggesting that perhaps the trait anxiety-X-T reactivity interaction observed in Study 1 may be specific to winners - whereas a simple main effect of $T$ reactivity on aggressive behavior would emerge among losers. However, it also remains possible that a trait anxiety-X-T reactivity interaction may also emerge among losers of competition. Thus, we made no specific predictions concerning the potential context specificity (i.e., win vs. loss) of the trait anxiety-X-T reactivity interaction.

\section{Study 2}

Materials and Methods

\section{Participants}

237 participants ( $52 \%$ women, $M_{\text {age }}=21.73, S D=4.66$ ) were recruited from the Wayne State University Research Participation Pool and were awarded partial credit and a \$5 honorarium. Trait anxiety, T reactivity, and aggression data were available for 224 participants (52.6\% women).

\section{Procedure}

Participants first completed a consent form, after which they completed a small battery of self-report measures including the STAI. Again, trait anxiety scores were calculated by summing responses across items and dividing by 20 to obtain a measure of mean anxiety (Cronbach's $\alpha=.88, M=1.97, S D=.41$ ). Next, participants provided a $1-2 \mathrm{ml}$ saliva sample (pre-competition) via passive drool into a polystyrene culture tube. Participants were then randomly assigned to experience a string of victories or defeats (15 min duration) in an Xbox 360 Kinect video game (see Carré et al. 2013 for full

Table 2 Trait anxiety moderates the relationship between testosterone reactivity and aggressive behavior (Study 1)

\begin{tabular}{llllll}
\hline Predictor & $B$ & $S E$ & $t$ & $p$ & $95 \%$ CIs \\
\hline T reactivity & -.06 & .68 & -.08 & .93 & $-1.41,1.30$ \\
Trait anxiety & -34.65 & 32.81 & -1.06 & .29 & $-100.04,30.73$ \\
T reactivity X trait anxiety & -3.38 & 1.63 & -2.08 & .04 & $-6.62,-.14$ \\
\hline
\end{tabular}

Note: $T=$ testosterone 
details of the Xbox task). Afterwards, they completed a questionnaire assessing their thoughts about the video game followed by a second 1-2 ml saliva sample (postcompetition) $20 \mathrm{~min}$ after the onset of the video game competition. Saliva samples were stored at $-20 \mathrm{C}$ until assayed in house using commercially available enzyme immunoassay kits (DRG International). All samples were assayed in duplicate and the intra-assay CV was $11.72 \%$. Across high and low controls, the average inter-assay CV was $14.88 \%$.

After playing the Xbox game, participants performed the PSAP task and were told they were paired with another same-sex participant (actually a computer program). The task was exactly the same as Study 1, with the exception that participants performed 3 blocks of the task, each lasting $7 \mathrm{~min}$. We computed aggressive behavior using the same method as Study 1; regressing average aggressive responses onto average reward and average protection responses and saved the unstandardized residuals.

Analysis

Because our previous analyses with this same dataset indicated that $\mathrm{T}$ dynamics predicted aggression in men, but not women (see Carré et al. 2013), we split our analyses by participant sex. Consistent with Study 1, T reactivity was measured by regressing post-video game $\mathrm{T}$ concentrations onto pre-video game $\mathrm{T}$ concentrations and saving the unstandardized residuals. First, we examined the extent to which trait anxiety would moderate the effect of competition outcome on $\mathrm{T}$ reactivity. Moderated multiple regression analysis was performed using the PROCESS macro for SPSS (Hayes 2012; 2013; Model 1), where competition outcome was set as the IV, trait anxiety as the moderator, and T residuals as the DV in Model 1. Trait anxiety was mean-centered prior to computing the interaction term and simple slopes were calculated at \pm 1 SDs. Also, because participants were randomly assigned to experience a string of victories or defeats, we examined the extent to which competition outcome would interact with $\mathrm{T}$ reactivity and trait anxiety to predict aggressive behavior (i.e., outcome-X-T reactivity-X- trait anxiety interaction; Model 3 in PROCESS macro). Both $\mathrm{T}$ reactivity and aggression were computed in the same way as Study 1.

\section{Results and Discussion}

Does Trait Anxiety Moderate the Relationship Between Competition Outcome and T Reactivity? Men: As previously reported (Carré et al. 2013), there was a main effect of competition outcome, whereby winners $(M=13.96, S E=2.79)$ had higher T concentrations relative to losers $(M=-5.07, S E=2.93)$. In contrast, there was no effect of trait anxiety on T reactivity $(p=.96)$ and there was no competition outcome $\mathrm{X}$ trait anxiety interaction ( $p=.41$; see Fig. 2 ).

Women: Results indicated no significant main effect of trait anxiety or competition outcome $(p s>.31)$ and no competition outcome-X-trait anxiety interaction $(p=.79)$.

Does Trait Anxiety Moderate the Relationship Between $T$ Reactivity and Aggression? Men: As previously reported (Carré et al. 2013), there was a significant bivariate correlation between T reactivity and aggression $(r=.27, p=.006)$. In contrast, there were no relationships between $\mathrm{T}$ reactivity and trait anxiety $(r=.04, p=.66)$, or between trait anxiety and aggression $(r=.05, p=.59)$. Consistent with results from 
Fig. 2 Male winners have elevated testosterone concentrations relative to losers, irrespective of individual differences in trait anxiety (Study 2)

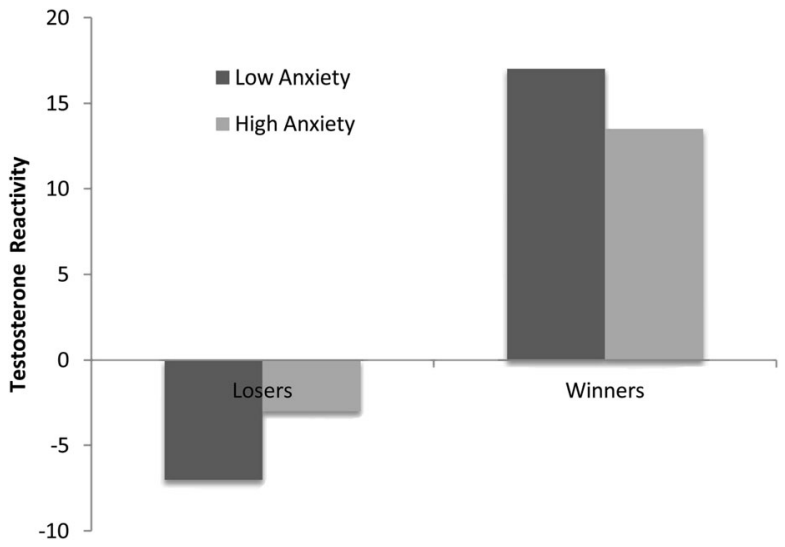

Study 1 , we found a significant $\mathrm{T}$ reactivity-X-trait anxiety interaction $(B=-2.24$, $\left.S E=1.03, p=.03,95 \% \mathrm{CIs}=-4.28,-.20, \mathrm{r}_{\text {partial }}=-.21\right)$. Notably, the effect size of this hypothesized interaction $\left(r_{\text {partial }}=-.21\right)$ was similar in magnitude to that of Study 1 $\left(r_{\text {partial }}=-.18\right)$. Simple slopes analyses indicated that $\mathrm{T}$ reactivity was positively correlated with aggressive behavior in men with low trait anxiety $(-1 \mathrm{SD} ; B=1.07, S E=.41$, $p=.01,95 \% \mathrm{CIs}=.26,1.87)$, but not high trait anxiety $(+1 \mathrm{SD} ; B=.23, S E=.33, p=.50$, $95 \% \mathrm{CIs}=-.44, .89)$. See Fig. 3 and Tables 3 and 4. Although we did not observe a moderating effect of competition outcome, we decided to explore the relationship between $\mathrm{T}$ reactivity and aggressive behavior separately for winners and losers scoring low $(-1 \mathrm{SD})$ and high $(+1 \mathrm{SD})$ in trait anxiety using simple slopes. For winners, $\mathrm{T}$ reactivity was positively correlated with aggressive behavior in men with low trait anxiety $(-1 \mathrm{SD} ; B=1.69, \mathrm{SE}=.88, p=.057,95 \% \mathrm{CIs}=-.05,3.43)$, but not high anxiety $(+1 \mathrm{SD} ; B=-.68, S E=.87, p=.44,95 \% \mathrm{CIs}=-2.42,1.05)$. For losers, T reactivity was positively correlated with aggressive behavior in men with low trait anxiety $(-1 \mathrm{SD}$, $B=1.32, S E=.66, p=.048,95 \% \mathrm{CIs}=.013,2.62)$, but not high trait anxiety ( $+1 \mathrm{SD}$, $B=.40, S E=.31, p=.21,95 \% \mathrm{CIs}=-.23,1.02)$. Thus, as suggested by the non-

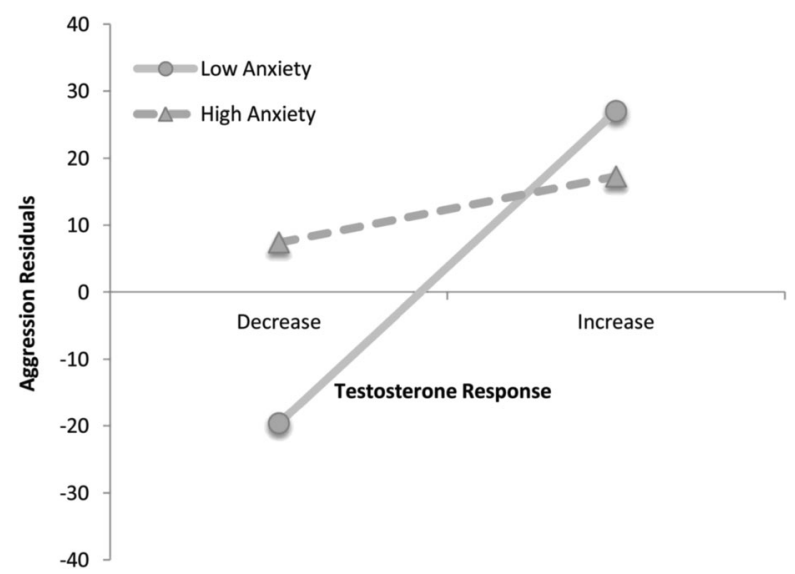

Fig. 3 Trait anxiety moderates the relationship between testosterone reactivity and aggressive behavior in men (Study 2). Note: Testosterone and trait anxiety are plotted at $+/-1 \mathrm{SD}$ from the mean 
Table 3 Descriptive statistics for testosterone in Study 2

\begin{tabular}{|c|c|c|c|c|c|c|c|}
\hline & \multirow[t]{2}{*}{ Time } & \multicolumn{2}{|c|}{ Winners and losers } & \multicolumn{2}{|c|}{ Winners } & \multicolumn{2}{|l|}{ Losers } \\
\hline & & M & SD & M & SD & M & SD \\
\hline \multirow[t]{2}{*}{ Men } & 1 & 93.83 & 39.99 & 92.42 & 37.39 & 95.17 & 42.60 \\
\hline & 2 & 86.91 & 38.63 & 95.21 & 43.34 & 78.76 & 31.67 \\
\hline \multirow[t]{2}{*}{ Women } & 1 & 41.09 & 20.59 & 42.47 & 19.08 & 39.71 & 22.07 \\
\hline & 2 & 36.23 & 17.66 & 35.68 & 17.41 & 36.77 & 18.02 \\
\hline
\end{tabular}

significant 3-way interaction - the trait anxiety-x-T reactivity interaction was essentially the same for winners and losers.

In addition to the trait anxiety-X-T reactivity interaction, we also observed a significant trait anxiety-X-competition outcome interaction $(B=76.33, S E=38.17$, $\left.p=.049,95 \% \mathrm{CIs}=.51,152.15, \mathrm{r}_{\text {partial }}=.18\right)$. Here, winners were more aggressive than losers in high anxious men $(+1 \mathrm{SD} ; B=35.50, S E=15.98, p=.029,95 \% \mathrm{CIs}=3.80$, 67.20), but not low anxious men ( $-1 \mathrm{SD} ; B=8.61, S E=18.00, p=.63,95 \%$ CIs $=-27.09,44.32$ ).

Women: There were no main effects of $\mathrm{T}$ reactivity, trait anxiety, or competition outcome (all $p \mathrm{~s}>.27$ ). Furthermore, there were no significant anxiety-X-T reactivity, anxiety-X-outcome, or anxiety-X-T reactivity-X-outcome interactions (all $p \mathrm{~s}>.25$ ).

Results from Study 2 are consistent with the findings from Study 1. Moreover, we extend the results from Study 1 by demonstrating that the T reactivity-X-trait anxiety interaction was similar in winners and losers and that this effect was specific to men. Our results also indicate that trait anxiety moderates the effect of competition outcome on subsequent aggression in men. Specifically, winning was associated with increased aggression, but only in men scoring relatively high in trait anxiety. The latter finding was not expected and future research will be needed to verify the robustness of this effect.

Table 4 Trait anxiety moderates the relationship between testosterone reactivity and aggressive behavior in men (Study 2)

\begin{tabular}{llllll}
\hline Predictor & $B$ & $S E$ & $t$ & $p$ & $95 \%$ CIs \\
\hline T reactivity & .69 & .33 & 2.10 & .039 & $.04,1.24$ \\
Trait anxiety & 25.08 & 18.66 & 1.34 & .18 & $-11.99,62.15$ \\
Outcome & .39 & 14.80 & .03 & .98 & $-29.01,29.78$ \\
T reactivity X trait anxiety & -2.24 & 1.03 & -2.18 & .032 & $-4.28,-.20$ \\
T reactivity X outcome & -.35 & .67 & -.52 & .60 & $-1.68, .98$ \\
Trait anxiety X outcome & 76.33 & 38.17 & 2.00 & .049 & $.51,152.15$ \\
T reactivity X trait anxiety X outcome & -2.03 & 2.12 & -.96 & .34 & $-6.25,2.19$ \\
\hline
\end{tabular}

Note: $T=$ testosterone 


\section{General Discussion}

There is a large body of evidence indicating that $\mathrm{T}$ concentrations rise in the context of competitive interactions and remain elevated in winners relative to losers (see Archer 2006 for meta-analysis). Functional interpretations of such findings suggest that acute changes in $\mathrm{T}$ within the context of competition may serve to fine-tune ongoing and/or future social behavior. Indeed, several recent studies support such a functional role of competition-induced T dynamics (Mehta and Josephs 2006; Carré and McCormick 2008; Carré et al. 2009, 2013, 2014a, b; Geniole et al. 2013; Apicella et al. 2014; Zilioli and Watson 2014). Nevertheless, not all people who demonstrate a rise in $\mathrm{T}$ during competition exhibit heightened aggressive behavior in subsequent interactions, and it is likely that there are several factors that moderate the relationship between context dependent changes in $\mathrm{T}$ and aggressive behavior. Here, across two studies, we found that trait anxiety moderated the relationship between $\mathrm{T}$ responses to competition and aggressive behavior in men. Specifically, T responses to competition were positively correlated with subsequent aggression, but only in men scoring relatively low on a dispositional measure of anxiety. These findings highlight the importance of considering individual difference factors when attempting to characterize neuroendocrine mechanisms underlying human aggression.

Our findings are consistent with recent evidence examining the effect of power priming on risk-taking and mate-seeking behavior. In their work, Maner et al. (2012) found that priming power increased subsequent risk-taking and mate-seeking behavior, but only among men with relatively low dispositional levels of anxiety. Notably, powerful postures have also been shown to increase both $\mathrm{T}$ concentrations and risktaking behavior (Carney et al. 2010, but see Ranehill et al. in press) and assigning participants to powerful leadership positions also increases T concentrations (Akinola and Mendes 2013). Thus, it is possible that the Maner et al. (2012) power priming manipulation increased $\mathrm{T}$ concentrations, which in turn modulated subsequent risktaking and mate-seeking behavior, but only in low anxious men.

Although work in animal models indicates that trait anxiety modulates both $\mathrm{T}$ reactivity to threat and aggressive behavior (Veenema et al. 2007), we found no relationship between trait anxiety and $\mathrm{T}$ reactivity to competition or between trait anxiety and aggressive behavior on the PSAP. A key difference between our study and the rat study is that we assessed normal variation in trait anxiety, whereas the research by Veenema et al. (2007) was based on creating extreme groups of rats that were either high or low on anxiety. Thus, we cannot rule out the possibility that extreme levels of trait anxiety may be related to variation in $\mathrm{T}$ reactivity to competition and/or aggressive behavior. In addition, previous work found that trait anxiety moderates the relationship between competition outcome and $\mathrm{T}$ reactivity in a small sample of young men $(N=23$, Maner et al. 2008). Here, using a much larger sample of men $(N=106)$, we found no support for a moderating effect of trait anxiety on $T$ reactivity to winning and losing. Specifically, winning was associated with increased $\mathrm{T}$ concentrations relative to losing, irrespective of one's level of trait anxiety (see Fig. 2).

Perhaps the moderating effect of trait anxiety on the relationship between $\mathrm{T}$ reactivity and aggression can be explained by different cognitive appraisals during the PSAP task between those with low versus high anxiety. When confronted with social provocation that requires action, a person will evaluate their own resources against the 
demands of the situation and the resulting assessment determines if the situation is perceived as a challenge or a threat (see Seery 2011 for a review). Research has also shown that low anxious people appraise stressful situations as a challenge whereas high anxious people appraise stressful situations as a threat (Jerusalema 1990). High anxiety also reduced risk -taking behaviors in a group of clinically anxious patients by increasing perceptions of potential threat, amplifying negative attitudes towards consequences, and promoting risk avoidance (Giorgetta et al. 2012). In the present study, participants with low dispositional anxiety, coupled with the endocrine response of an increase in T, may assess their resources as surpassing the demands of the PSAP task and therefore view it as a challenge, eliciting a more competitive or aggressive behavioral response. Conversely, those with high anxiety may assess the PSAP as social provocation for which they lack sufficient resources, and therefore retreat and do not engage their partner. As such, a rise in T only predicts an aggressive behavioral response to provocation among individuals who process the provocation during the PSAP task as challenging (i.e. low anxious people). For those who process the provocation as a threat, the rise in $\mathrm{T}$ does not translate into an aggressive behavioral response. Although future research examining cognitive appraisals and aggressive behavior is needed to test such hypotheses, this work is consistent with previous studies examining social anxiety and aggressive behavior (DeWall et al. 2010). Specifically, DeWall and colleagues found that socially anxious individuals are more likely to perceive hostility from others, yet are unlikely to engage in overt aggressive behavior.

DeWall et al. (2010) also reasoned that socially anxious individuals may be less aggressive because they suppress anger. Therefore, anxious individuals that experience a rise in $\mathrm{T}$ in the face of competition may experience aggressive impulses, but suppress the urge to aggress against others. Future work is needed to assess the associations between $\mathrm{T}$ reactivity, anxiety, and aggressive impulses, in addition to aggressive behavior.

The importance of considering individual difference factors as moderators of hormone/behavior relationships is highlighted by a recent surge of research on oxytocin (OT) administration. There is now a growing body of evidence indicating that effects of OT on cognitive and behavioral processes depend critically on social-contextual and individual difference factors (see Bartz et al. 2011 for a review). There is also a wealth of research using single $\mathrm{T}$ administration protocols, mainly in women (see Bos et al. 2012), but also recently implemented in men (Goetz et al. 2014). The findings from the current studies indicate that it will be important to assess the extent to which trait anxiety moderates the effect of $\mathrm{T}$ administration on human aggressive behavior - and other behavioral traits linked to T (e.g., competitive motivation, affiliative behavior, trust ratings, etc.).

There are some limitations of the current studies that should be discussed. First, we utilized a competitive task that involves physical activity. It is known that physical activity can potentiate $T$ release (Kraemer and Ratamess 2005), and thus, the extent to which competition and/or physical activity contributes to the pattern of neuroendocrine responses cannot be determined. Future work should attempt to verify our findings using a competitive task that does not involve physical activity (e.g., Tetris; see Zilioli and Watson 2014). Second, our findings suggest that the moderating effect of trait anxiety on the relationship between $\mathrm{T}$ reactivity and aggression is specific to men (Study 2). Notably, men are much more inclined to play video games that involve 
physical competition (e.g., sports, fighters, shooters, and racing games), whereas women are more inclined to play video games that are traditional (e.g., puzzle games, card/dice games) and/or involve imagination (e.g., fantasy games or adventure games; Greenberg et al. 2010). Thus, we cannot rule out the possibility that having women engage in video games that are more appealing to them (e.g., traditional or imagination) would elicit stronger effects on neuroendocrine function and perhaps yield significant relationships between $\mathrm{T}$ reactivity and aggressive behavior.

In summary, we found that trait anxiety moderates the effect of $\mathrm{T}$ reactivity on aggressive behavior but only in men with low dispositional levels of anxiety. As expected, this response was sex specific, and was not moderated by competition outcome. These findings highlight the importance of considering the role of individual difference factors as potential moderators of hormone-behavior relationships in humans.

\section{References}

Akinola, M., \& Mendes, W. B. (2013). It's good to be the king: neurobiological benefits of higher social standing. Social Psychological and Personality Science, 5, 43-51.

Apicella, C. L., Dreber, A., \& Mollerstrom, J. (2014). Salivary testosterone change following monetary wins and losses predicts future financial risk-taking. Psychoneuroendocrinology, 39, 58-64.

Archer, J. (2006). Testosterone and human aggression: an evaluation of the challenge hypothesis. Neuroscience and Biobehavioral Reviews, 30, 319-345.

Archer, J., Graham-Kevan, N., \& Davies, M. (2005). Testosterone and aggression: a reanalysis of book, Starzyk, and Quinsey's (2001) study. Aggression and Violent Behavior, 10(2), 241-261.

Bartz, J. A., Zaki, J., Bolger, N., \& Ochsner, K. N. (2011). Social effects of oxytocin in humans: context and person matter. Trends in Cognitive Sciences, 15(7), 301-309.

Bos, P. A., Panksepp, J., Bluthéc, R.-M., \& van Honka, J. (2012). Acute effects of steroid hormones and neuropeptides on human social-emotional behavior: a review of single administration studies. Frontiers in Neuroendocrinology, 33(1), 17-35.

Carney, D. R., Cuddy, A. J. C., \& Yap, A. J. (2010). Power posing: brief nonverbal displays affect neuroendocrine levels and risk tolerance. Psychological Science, 20, 1-6.

Carré, J. M., \& McCormick, C. M. (2008). Aggressive behavior and change in salivary testosterone concentrations predict willingness to engage in a competitive task. Hormones and Behavior, 54(3), 403-409.

Carré, J. M., Putnam, S. K., \& McCormick, C. M. (2009). Testosterone responses to competition predict future aggressive behaviour at a cost to reward in men. Psychoneuroendocrinology, 34(4), 561-570.

Carré, J. M., McCormick, C. M., \& Hariri, A. R. (2011). The social neuroendocrinology of human aggression. Psychoneuroendocrinology, 36(7), 935-944.

Carré, J. M., Campbell, J. A., Lozoya, E., Goetz, S. M., \& Welker, K. M. (2013). Changes in testosterone mediate the effect of winning on subsequent aggressive behaviour. Psychoneuroendocrinology, 38(10), 2034-2041.

Carré, J.M., Iselin, A.M., Welker, K.M., Hariri, A.R., \& Dodge, K.A.(2014a). Testosterone reactivity to provocation mediates the effect of early intervention on aggressive behavior. Psychological Science, 25, 1140-1146.

Carré, J. M., Baird-Rowe, C. D., \& Hariri, A. R. (2014b). Testosterone responses to competition predict decreased trust ratings of emotionally neutral faces. Psychoneuroendocrinology, 49, 79-83.

Cherek, D.R., Tcheremissine, O.V., \& Lane, S.D. (2006). Psychopharmacology of human aggression: laboratory and clinical studies. Biology of aggression, 424-446.

DeWall, C. N., Baumeister, R. F., Stillman, T. F., \& Gailliot, M. T. (2007). Violence restrained: effects of selfregulation and its depletion on aggression. Journal of Experimental Social Psychology, 43, $62-76$.

DeWall, C. N., Buckner, J. D., Lambert, N. M., Cohen, A. S., \& Fincham, F. D. (2010). Bracing for the worst, but behaving the best: social anxiety, hostility, and behavioral aggression. Journal of Anxiety Disorders, 24(2), 260-268.

Fuxjager, M. J., Forbes-Lorman, R. M., Coss, D. J., Auger, C. J., Auger, A. P., \& Marler, C. A. (2010). Winning territorial disputes selectively enhances androgen sensitivity in neural pathways related to 
motivation and social aggression. Proceedings of the National Academy of Sciences USA, 107(27), 12393-12398.

Geniole, S. N., Busseri, M. A., \& McCormick, C. M. (2013). Testosterone dynamics and psychopathic personality traits independently predict antagonistic behavior towards the perceived loser of a competitive interaction. Hormones and Behavior, 64(5), 790-798.

Giorgetta, C., Grecucci, A., Zuanon, S., Perini, L., Balestrieri, M., Bonini, N., Sanfey, A. G., \& Brambilla, P. (2012). Reduced risk-taking behavior as a trait feature of anxiety. Emotion, 12(6), 1373-1383.

Gleason, E. D., Fuxjager, M. J., Oyegbile, T. O., \& Marler, C. A. (2009). Testosterone release and social context: when it occurs and why. Frontiers in Neuroendocrinology, 30(4), 460-469.

Goetz, S. M., Tang, L., Thomason, M. E., Diamond, M. P., Hariri, A. R., \& Carré, J. M. (2014). Testosterone rapidly increases neural reactivity to threat in healthy men: a novel two-step pharmacological challenge paradigm. Biological Psychiatry, 76(4), 324-331.

Greenberg, B. S., Sherry, J., Lachlan, K., Lucas, K., \& Holmstrom, A. (2010). Orientations to video games among gender and age groups. Simulation \& Gaming, 41(2), 238-259.

Hayes, A.F. (2012). PROCESS: A versatile computational tool for observed variable mediation, moderation, and conditional process modeling [White paper]. Retrieved from http://www.afhayes.com/public/ process2012.pdf

Hayes, A. F. (2013). Introduction to mediation, moderation, and conditional process analysis. New York: The Guilford Press.

Jerusalema, M. (1990). Temporal patterns of stress appraisals for high- and low-anxious individuals. Anxiety Research, 3(2), 113-129.

Ketterson, E. D., \& Nolan, V. (1992). Hormones and life histories: an integrative approach. The American Naturalist, 140, S33-S62.

Kraemer, W. J., \& Ratamess, N. A. (2005). Hormonal responses and adaptations to resistance exercise and training. Sports Medicine, 35, 339-361.

Maner, J. K., Miller, S. L., Schmidt, N. B., \& Eckel, L. A. (2008). Submitting to defeat: social anxiety, dominance threat, and decrements in testosterone. Psychological Science, 19(8), 764-768.

Maner, J. K., Gailliot, M. T., Menzel, A. J., \& Kunstman, J. W. (2012). Dispositional anxiety blocks the psychological effects of power. Personality and Social Psychology Bulletin, 38(11), 1383-1395.

Mazur, A. (1985). A biosocial model of status in face-to-face primate groups. Social Forces, 64(2), 377-402.

McGlothlin, J. W., Jawor, J. M., \& Ketterson, E. D. (2007). Natural variation in a testosterone-mediated tradeOff between mating effort and parental effort. The American Naturalist, 170(6), 864-875.

Mehta, P. H., \& Josephs, R. A. (2006). Testosterone change after losing predicts the decision to compete again. Hormones and Behavior, 50(5), 684-692.

Oliveira, R. F. (2009). Social behavior in context: hormonal modulation of behavioral plasticity and social competence. Integrative and Comparative Biology, 49(4), 423-440.

Ranehill, E., Dreber, A., Johannesson, M., Leiberg, S., Sul, S., \& Weber, R. (in press). Assessing the robustness of power posing: no effects on hormones and risk taking in a large sample of men and women. Psychological Science.

Seery, M. D. (2011). Challenge or threat? cardiovascular indexes of resilience and vulnerability to potential stress in humans. Neuroscience and Biobehavioral Reviews, 35, 1603-1610.

Simon, N., \& Lu, S. (2006). Androgens and aggression. In R. J. Nelson (Ed.), Biology of aggression (pp. 211230). New York: Oxford University Press.

Spielberger, C. D., Gorsuch, R. L., Lushene, R., Vagg, P. R., \& Jacobs, G. A. (1983). Manual for the state-trait anxiety inventory. Palo Alto: Consulting Psychologists Press.

Trainor, B. C., Bird, I. M., \& Marler, C. A. (2004). Opposing hormonal mechanisms of aggression revealed through short-lived testosterone manipulations and multiple winning experiences. Hormones and Behavior, 45(2), 115-121.

Veenema, A. H., Torner, L., Blume, A., Beiderbeck, D. I., \& Neumann, I. D. (2007). Low inborn anxiety correlates with high intermale aggression: link to ACTH response and neuronal activation of the hypothalamic paraventricular nucleus. Hormones and Behavior, 51(1), 11-19.

Wingfield, J. C., Hegner, R. E., Dufty, A. M., Jr., \& Ball, G. F. (1990). The 'challenge hypothesis': theoretical implications for patterns of testosterone secretion, mating systems, and breeding strategies. The American Naturalist, 136, 829-846.

Zilioli, S., \& Watson, N. V. (2014). Testosterone across successive competitions: evidence for a winner effect in humans? Psychoneuroendocrinology, 47, 1-9. 